\title{
A RATIO-DEPENDENT PREDATOR-PREY MODEL WITH STRONG ALLEE EFFECT IN THE PREY AND AN ALTERNATIVE FOOD SOURCE FOR THE PREDATOR
}

\author{
Alakes Maiti ${ }^{1 *}$, Rita Paul$^{2}$, Shariful Alam ${ }^{3}$ \\ ${ }^{1}$ Department of Mathematics, Vidyasagar Evening College, Kolkata-700006, India, alakeshmaity@hotmail.com \\ ${ }^{* 1}$ Corresponding Author \\ ${ }^{2}$ Department of Mathematics, Indian Institute of Engineering Science and Technology, Shibpur, Howrah - 711103, \\ India \\ ${ }^{3}$ Department of Mathematics, Indian Institute of Engineering Science and Technology, Shibpur, Howrah - 711103, \\ India, salam50in@yahoo.co.in
}

\begin{abstract}
This paper aims to study the dynamical behaviours of a ratio-dependent predator-prey model, where the prey is subject to strong Allee effect, and the predator has an alternative food source. Positivity and boundedness of the system are discussed. Some extinction criteria are derived. Stability analysis of the equilibrium points is presented including some global results. Numerical simulations are carried out to validate our analytical findings. Implications of our analytical and numerical findings are discussed critically.
\end{abstract}

Keywords: Predator-Prey, Allee Effect, Extinction, Stability.

\section{INTRODUCTION}

Nowadays the study of predator-prey relationship has become an extremely important part of ecology. In this relationship, one species serves as a food for the other. It is true that the preys always try to develop the methods of evasion to avoid being eaten. However, it is certainly not true that a predator-prey relationship is always harmful for the preys, it might be beneficial to both. Further, such a relationship often plays an important role to keep ecological balance in nature.

Mathematical modelling of predator-prey interaction was started in 1920s. Interestingly, the first predator-prey model in the history of theoretical ecology was developed independently by Alfred James Lotka (a US physical chemist) and Vito Volterra (an Italian mathematician) [30, 48]. Subsequently, this model has been used as a machine to introduce numerous mathematical and practical concepts in theoretical ecology. Many refinements of the Lotka-Volterra model have also been made to overcome the shortcomings of the model and to get better insights of predator-prey interactions. In the last five or six decades, a number of predator-prey models are developed and systematically cultured in literature. However, urge for incorporating many parameters of real systems had been felt day by day.

If we summarize the basic considerations behind the modelling of predator-prey systems, it would be evident that the most crucial elements of predator-prey models are the choices of growth function of the prey and functional response of the predator.
It has long been recognized that the famous logistic growth function has the capability of describing individual population growth. The function is introduced in 1838 by the Belgian mathematician Pierre Francois Verhulst [47] and later it is rediscovered in 1920 by American biologists Reymon Pearl and Lowell Reed [39]. If $X(T)$ denotes the population density at time $T$, then the logistic growth equation is given by

$\frac{d X}{d T}=r X\left(1-\frac{X}{K}\right)$

where $r$ is the intrinsic per capita growth rate and $K$ is the carrying capacity of the environment. The logic behind this is very simple. As the resources (e.g., space, food, and essential nutrients) are limited, every population grows into a saturated phase from which it cannot grow further; the ecological habitat of the population can carry just so much of it and no more[83]. This suggests that the per capita growth rate is a decreasing function of the size of the population, and reaches zero as the population achieved a size $K$ (in the saturated phase). Further, any population reaching a size that is above this value will experience a negative growth rate. The term $-r X^{2} / K$ may also be regarded as the loss due to intraspecific competition. Although logistic growth function became extremely popular, but, in real life situations, researchers found many evidences where the populations show a reverse trend in low population density $[18,16,36,12,19$, 42]. This phenomenon of positive density dependence of population growth at low densities is known as the Allee effect $[45,19]$. 
The phenomenon of Allee effect is named after the US Behavioral scientist Warder Clyde Allee (although Allee never used the term 'Allee effect'). Allee described this concept in three of his papers $[3,4,5]$. Actually, the term 'Allee effect' was introduced by Odum [38]. Since the late eighties of the 20th century, the concept gained importance but there were necessity of clear-cut definitions and clarification of concepts. The necessity

was fulfilled when three reviews by Stephens et al. [45], Courchamp et al. [18], Stephens and Sutherland [44]. There are many reasons for Allee effect, such as difficulty in mate finding, reduced antipredator vigilance, problem of environmental conditioning, reduced defense against predators, and many others (for thorough reviews, see references $[12,19])$. The Allee effect can be divided into two main types, depending on how strong the per capita growth rate is depleted at low population densities. These two types are called the strong Allee effect $[46,50,51]$ or critical depensation [14, 15, 28], and the weak Allee effect [45, 49] or noncritical depensation [14, 15,28]. Usually, the Allee effect is modelled by a growth equation of the form

$\frac{d X}{d T}=r X\left(1-\frac{X}{K}\right)\left(\frac{X}{K_{0}}-1\right)$,

where $X(T)$ denotes the population density at time $T, r$ is the intrinsic per capita growth rate, and $K$ is the carrying capacity of the environment. Here $0<K_{0} \ll K$. When $K_{0}>0$ and the population size is below the threshold level $K_{0}$, then the population growth rate decreases $[9,17,22$, 27], and the population goes to extinction. In this case, the equation describes the strong Allee effect [46, 50, 51]. On the contrary, the description of weak Allee effect is also available (see references [50, 23]). In this paper, we are concerned with strong Allee effect. The above growth is often said to have a multiplicative Allee effect. There is another mathematical form of the growth function featuring the additive Allee effect. In this paper, we are not interested in additive Allee effect (interested readers might see the works of Aguirre et al. [1, 2]). A comparison of the logistic growth function of (1.1) and the function representing Allee effect in equation (1.2) can be found in [35].

Let us now turn our attention from the individual growth of the prey to the interaction of the prey and its predator. The function that describes the number of prey consumed per predator per unit time for given quantities of prey and predator is known as the functional response or trophic function[11,81]. Depending upon the behaviour of populations, more suitable functional responses have been developed as a quantification of the relative responsiveness of the predation rate to change in prey density at various populations of prey. In this connection, Holling family of functional responses are the most focused [25, 26]. The Holling type-I functional response (or the Lotka-Volterra functional response) is given by $F(X)=$ $\beta X$, where $X(T)$ is the prey density at time $T$ and $\beta>0$ is a constant. The Holling type-II (or Michaelis-Menten) functional response has become extremely popular. The
type-II functional response includes the fact that a single individual can feed only until the stomach is not full, and so a saturation function would be better to describe the intake of food. This is similar to the concept of the law of diminishing returns borrowed from operations research, via a hyperbola rising up to an asymptotic value. In other words, the functional response would be of the following form

$\beta X$

$F(X)=\alpha+X$,

where $X(T)$ is the prey density at time $T, \beta>0$ is the maximal growth rate of the predator and $\alpha>0$ is the half saturation constant. Although these functional responses have served as basis for a very large literature in predatorprey theory (see [37, 43, 32], and references therein); but there should be no denying that, in many situations, the predator density could have a direct effect on functional response. In 1989, Arditi and

Ginzburg [6] labelled those functional responses, which depends on prey-density only, as "prey-dependent" functional responses. Afterwards, a number of "predator-dependent" models have been proposed, the most widely known being those of Hassell and Varley [24], DeAngelis et al. [20], Beddington [10] and Arditi and Akcakaya [7]. A predatordependent functional response, which is a function of the ratio of the prey and predator, is known as a ratio-dependent functional response. Arditi and Ginzburg [6] introduced a Michaelis-Menten type ratio-dependent functional response of the form

$F(X / Y)=\frac{\beta(X / Y)}{\alpha+(X / Y)}=\frac{\beta X}{\alpha Y+X}$,

Where X,Y stand for densities of prey and predator respectively. The positive constants $\beta$ and $\alpha$ are the maximal growth rate of the predator and the half capturing saturation constant, respectively. Predator-prey models with such ratiodependent functional response are strongly supported by numerous field and laboratory experiments (see [33] and references therein). Detailed arguments on the merits of ratio-dependent model in comparison with other models may be found in the works of Berezovskaya et al. [13] and Arditi et al. [8].

Once again, we want to go back to the question of individual population growth. Even if a population is not susceptible to Allee effect, it is easy to understand that the logistic growth function might not be capable of explaining the growth of each population. Obviously, several modifications of logistic growth function have been proposed, whilst retaining the sigmoid and asymptotic property of the logistic curve. In 1959, Richards [41] suggested the growth equation in the following form:

$\frac{d X}{d T}=r X\left[1-\left(\frac{X}{K}\right)^{\delta}\right]$, 
where $\delta$ is positive exponent and it describes the degree of intraspecific competition.

In this paper, we have considered a predator-prey model, where the prey is vulnerable to strong Allee effect. In this situation, it is quite natural that predator must try to manage some alternative food source. This is obviously not very easy, and therefore, there must be serious intraspecific competition among predators. From this viewpoint, we have considered a growth function of the form (1.5) and a Michaelis-Menten type ratio-dependent functional response for predators.

The rest of the paper is structured as follows. In section 2, we present a brief sketch of the construction of the model and the biological relevance of it. In section 3 , positivity and boundedness of the basic deterministic model is discussed. Some theorems on extinction of the populations are presented in section 4. Section 5 deals with the equilibrium points and their stability analysis. In section 7 , computer simulation of a variety of numerical solutions of the system is presented. Section 8 contains the general discussions on the results and biological implications of our mathematical findings.

\section{THE MATHEMATICAL MODEL}

Before we introduce the mathematical model, let us describe the basic assumptions that we made to formulate it.

1. The biological system we consider is composed of a single prey population whose density at time $T$ is denoted by $X$. The density of the predator at time $T$ is denoted by $Y$.

2. We consider a multiplicative Allee effect in prey population growth.

3. As the prey is subject to Allee effect, it is assumed that the predator manage some additional food source (for which there is serious intraspecific competition among them), and there is mutual interference among the predators for the prey $X$ also. Therefore, we assume that the predator follows a modified logistic growth of the form (1.5) and the predator consumes the prey with a Michaelis-Menten type ratio-dependent functional response.

The above considerations motivate us to introduce the following predator-prey model under the framework of the following set of nonlinear ordinary differential equations:

$$
\begin{aligned}
& \frac{d X}{d T}=r X\left(1-\frac{X}{K}\right)\left(\frac{X}{K_{0}}-1\right)-\frac{\alpha X Y}{c_{1} Y+X}, X(0)>0 \\
& \frac{d Y}{d T}=s Y\left[1-\left(\frac{Y}{L}\right)^{\delta}\right]+\frac{\alpha \lambda X Y}{c_{1} Y+X}, Y(0)>0
\end{aligned}
$$

We define the right side of each of the equation in (2.1) as 0 when $(X, Y)=(0,0)$. Here $r$ is the intrinsic growth rate, $K$ is the carrying capacity, and $K_{0}$ is the Allee threshold for the prey. $s$ is the intrinsic growth rate of the predator, $L$ its carrying capacity, and $\delta$ represents the degree of intraspecific competition. The parameter $\alpha$ denotes the maximal growth rate of the predator; and $\gamma$ is the biomass conversion rate of the predator. The parameters $c 1$ is the half saturation constant. We also assume that all parameters are positive.

To reduce the number of parameters, we use the following scaling

$$
\frac{X}{K}=x, \frac{Y}{L}=y, t=r \frac{K}{K_{0}} T .
$$

Then the system (2.1) becomes

$$
\begin{aligned}
& \frac{d x}{d t}=x(1-x)(x-m)-\frac{p x y}{c y+x}, x(0)>0 \\
& \frac{d y}{d t}=e y\left(1-y^{\delta}\right)+\frac{q x y}{c y+x}, y(0)>0
\end{aligned}
$$

Where

$$
\begin{aligned}
& m=\frac{K_{0}}{K}, p=\frac{\alpha K_{0} L}{r K^{2}}, c=\frac{c_{1} L}{K}, e=\frac{K_{0}}{r K}, q=\frac{\alpha \gamma K_{0}}{r K}, \text { and } \\
& \text { and } x(0)>0, y(0)>0, z(0)>0 .
\end{aligned}
$$

We notice that the right side of each of the equation in (2.2) is equal to 0 when $(x, y)=(0,0)$

\section{POSITIVITY AND BOUNDEDNESS}

Positivity and boundedness of a model guarantee that the model is biologically well behaved. It is easy to notice that the functions on the right side of each of the equations in (2.2) are continuously differentiable in $\mathrm{R}^{2}$. Therefore the solution of (2.2) with a positive initial condition exists and is unique. For positivity of the system (2.2), we have the following theorem.

Theorem 3.1 All solutions of the system (2.2) that start in $\mathrm{R}_{+}{ }^{2}$ remain positive forever.

The proof is simple and therefore it is omitted. Now we prove and useful result

Theorem $3.2 \lim \sup _{t \rightarrow \infty} x(t) \leq 1$.

Proof. Case-I. Let $x(0) \leq 1$. We claim that $x(t) \leq 1$ for all $t$ $\geq 0$.

If possible, assume that our claim is not Then it is possible to find two positive real numbers $t^{\prime}$ and $t^{\prime \prime}$ such that $x\left(t^{\prime}\right)=1$ and $x(t)>1$ for all $t \in\left(t^{\prime}, t^{\prime \prime}\right)$.

Now, for all $t \in\left(t^{\prime}, t^{\prime \prime}\right)$, we have from the first equation of (2.2)

$x(t)=x(0) \exp \left(\int_{0}^{t} \phi(x(s), y(s)) d s\right)$ 
$x(t)=x(0) \exp \left(\int_{0}^{t} \phi(x(s), y(s)) d s\right)<x(0)$

where $\phi(x(t), y(t))=(1-x(t))(x(t)-m)-\frac{p y(t)}{c y(t)+x(t)}$.

This implies that

$x(t)=x(0)\left[\exp \left(\int_{0}^{t^{\prime}} \phi(x(s), y(s)) d s\right)\right]\left[\exp \left(\int_{t^{\prime}}^{t} \phi(x(s), y(s)) d s\right)\right]$

$=x\left(t^{\prime}\right) \exp \left(\int_{t^{\prime}}^{t} \phi(x(s), y(s)) d s\right)$, for all $t \in\left(t^{\prime}, t^{\prime \prime}\right)$

since $\mathrm{m}<1$, we have $\phi(x(t), y(t))<0$ for all $t \in\left(t^{\prime}, t^{\prime \prime}\right)$.

Consequently, we have $x(t)<x\left(t^{\prime}\right)$, where $x\left(t^{\prime}\right)=1$.

This is contrary to the assumption that $x(t)>1$ for all $t \in\left(t^{\prime}, t^{\prime \prime}\right)$. Thus our claim is true.

Case-II . Let $x(0)>1$. We claim that $\lim \sup _{t \rightarrow \infty} x(t) \leq$ 1.

If possible, assume that this claim is false. Then $x(t)>1$ for all $t>0$. So $\varphi(x(t), y(t))<0$

( where $\varphi$ has the same expression as in Case-I); and consequently, we have from the first equation of (2.2) that

$x(t)=x(0) \exp \left(\int_{0}^{t} \phi(x(s), y(s)) d s\right)<x(0)$

Also from the first equation of $(2 . \overline{2})$, we obtain $\frac{1}{x} \frac{d x}{d t}<(x(0)-m) x(1-x)<0$, where $x(0)-m>0$.

This implies that $\limsup _{t \rightarrow \infty} x(t) \leq 0$, which is contradictory to our assumption. Therefore our claim is true.

From the above two cases, we have $\limsup _{t \rightarrow \infty} \mathrm{x}(\mathrm{t}) \leq 1$. The following theorem ensures the boundedness of the system (2.2).

Theorem 3.3 All solutions of the system (2.2) that starts in $R_{+}^{2}$ are uniformly bounded.

Proof. Let $W(x, y)=q x+p y$. Then

$\frac{d W}{d t} \leq q(1+m) x+p e y\left(1-y^{\delta}\right)$

For any $\mu>0$, we have

$\frac{d W}{d t}+\mu W \leq q(1+m+\mu) x+p e y\left(1+\mu-y^{\delta}\right)$.
Now for all $\mathrm{y}>0, \max \left\{y\left(1+\mu-y^{\delta}\right)\right\}=v$, where $v=\delta\left(\frac{1+\mu}{1+\delta}\right)^{\frac{1+\delta}{\delta}}$

Therefore, $\frac{d W}{d t}+\mu W \leq \sigma$, where $\sigma=q(1+m+\mu)+$ pev.

Applying the theory of differential inequalities, we obtain

$0 \leq W(x, y) \leq \frac{\sigma}{\mu}+\frac{W(x(0), y(0))}{e^{\mu t}}$.

and for $\mathrm{t} \rightarrow \infty, 0 \leq W \leq \frac{\sigma}{\mu}$.

Thus, all the solutions of (2.2) enter into the region $B=\left\{(x, y): 0 \leq W(x, y) \leq \frac{\sigma}{\mu}+\varepsilon\right.$, for any $\left.\varepsilon>0\right\}$.

Hence the theorem.

\section{EXTINCTION SCENARIOS}

In this section, we find some conditions for extinction of the prey. Here we use the symbols $x$ and $y$ to represent lim $\sup _{t \rightarrow \infty} x(t)$ and $\lim \inf _{t \rightarrow \infty} y(t)$, respectively. We frequently use the fact that $x \leq 1$, which is proved in Theorem 3.2.

It is quite obvious that if, after certain time, the prey population density lies below the Allee threshold (moreover there is attack of predator), then it is really impossible for the prey to survive. This fact is represented in mathematical terms in the following theorem.

Theorem 4.1 If $\bar{x}<\mathrm{m}$, then $\lim _{t \rightarrow \infty} x(t)=0$.

Proof: If possible,let $\lim _{t \rightarrow \infty} x(t)=\mu>0$.

The definition of $\bar{x}$ implies that for any $\varepsilon$ satisfying

$0<\varepsilon<m-\bar{x}$ there exists $\mathrm{t}_{\varepsilon}>0$ such that $\mathrm{x}(\mathrm{t})<\overline{\mathrm{x}}+\varepsilon$ for $\mathrm{t}>\mathrm{t}_{\varepsilon}$

Then for $\mathrm{t}>\mathrm{t}_{\varepsilon}$ we have from the first equation (2.2) that

$$
\begin{aligned}
x(t) & =x(0) \exp \left(\int_{0}^{t}\left\{\left(1-(x(s))(x(s)-m)-\frac{p y(s)}{c y(s)+x(s)}\right\} d s\right)\right. \\
& <x(0) \exp \left(\int_{0}^{t}(\bar{x}+\varepsilon-m) d s\right) \\
& <x(0) \exp \{-(m-\bar{x}-\varepsilon) t\} \rightarrow 0 \text { as } \mathrm{t} \rightarrow \infty,
\end{aligned}
$$

which is a cotradiction. This proves the theorem.

Remark. If the condition of the above theorem is satisfied, then the predator have no vital role in leading the prey to extinction, because the Allee effect is enough to do this (of course, the predator might expedite the process of extinction of the prey). The following theorem shows that the predator might also play a key role to prompt the prey to die out. 
Theorem 4.2 - If $\underline{\mathrm{y}}>\frac{1}{c}\left[\frac{2 c}{c m+p-2 c}-1\right]>0$, then $\lim _{t \rightarrow \infty} x(t)=0$.

Proof: if possible, let $\lim _{t \rightarrow \infty} x(t)=\mu>0$.

Since $\overline{\mathrm{x}} \leq 1$, for any $0<\varepsilon<1-\mathrm{m}$, there

exists $t_{\varepsilon}>0$ such that $y(t)>y-\varepsilon$ for $t>t_{\varepsilon}$.

From the definition of $\mathrm{y}$, it follows that, for any $o<\varepsilon^{\prime}<\frac{1}{c}\left[\frac{2 c}{c m+p-2 c}-1\right]$ there exists $t_{\varepsilon^{\prime}}>0$ such that $y(t)>y-\varepsilon^{\prime}$ for $t>t_{\varepsilon}$.

Then ,for $t>\max \left\{t_{\varepsilon}, t_{\varepsilon}\right\}$ we have from the first equation of (2.2) that

$$
\begin{aligned}
\frac{d x}{d t} & <x(1+\varepsilon-m)-\frac{p x y}{1+b x+c y} \\
& <x(1+\varepsilon-m)-\frac{p x y}{c y+(1+\varepsilon)} \\
& <x\left[(1+\varepsilon-m)-\frac{p}{c} \frac{c y}{c y+(1+\varepsilon)}\right], \\
& <x\left[(2-m)-\frac{p}{c}\left\{\frac{c y+(1+\varepsilon)-1-\varepsilon}{c y+1+\varepsilon}\right\}\right], \\
& <x\left\{2-m-\frac{p}{c}+\frac{1+b(1+\varepsilon)}{c y+1+\varepsilon}\right\}, \\
& <x\left\{2-m-\frac{p}{c}+\frac{1+\varepsilon}{c y+1+\varepsilon}\right\} \\
& <x\left\{2-m-\frac{p}{c}+\frac{2}{c(\underline{y}-\varepsilon)+1}\right\}<0 \text { [by the given condition], }
\end{aligned}
$$

Which implies that $\lim _{t \rightarrow \infty} x(t)=0$,

a contradiction. Hence the theorem is established.

\section{EQUILIBRIA AND THEIR STABILITY}

In this section, we find the equilibrium points of the system (2.2) and study their stability. The following lemma gives the boundary equilibrium points.

Lemma 5.1 The trivial equilibrium $E_{0}(0,0)$ of the system (2.2) always exists. There are two predator-free equilibrium points $E_{1}(1,0)$ and $E_{2}(m, 0)$, and one prey-free equilibrium point $E_{3}(0,1)$, each of which also exists unconditionally.

For the existence of the interior or coexistence equilibrium $E^{*}\left(x^{*}, y^{*}\right)$, we have the following lemma.

Lemma 5.2 If the equation

$c(\eta+p)(c \eta)^{\delta}+\left\{(c \eta)^{\delta}-(\eta+p)^{\delta} x^{\delta}\right\}+(1+q)(c \eta)^{\delta}=0$ has a positive root, $[$ where $\eta=c(1-x)(x-m)-p]$ then the first component $\mathrm{x}^{*}$ of the interior equlibrium $\mathrm{E}^{*}\left(x^{*}, y^{*}\right)$ exists, and it is positive root of (5.1).Further if $\left(1-x^{*}\right)\left(x^{*}-m\right)\{c(1-x)(x-m)-p\}>0$,

then $\mathrm{y}^{*}$ exist and is given by $\mathrm{y}^{*}=\frac{c\left(1-x^{*}\right)\left(x^{*}-m\right)}{c\left(1-x^{*}\right)\left(x^{*}-m\right)-p}$.

The system (2.2) cannot be linearized about E0 $(0,0)$. Therefore local stability of it is not studied. However, we can shed some light on the dynamics of the system (2.2) near origin giving some different logic. Since we are interested in the behavior near the origin,

we can take $\mathrm{x} \ll<1$ and $\mathrm{y} \ll<1$ and neglect terms in $\mathrm{x}$, $\mathrm{y}$ of order

2. We also notice

that $\frac{x}{c y+x} \leq 1$. Thus, near the origin, we have

$\frac{d x}{d t} \approx-m x$

$\frac{d y}{d t} \approx(e+q) y$.

This indicates that the origin is saddle.

The Jacobian matrix $J\left(E_{1}\right)$ at $\mathrm{E}_{1}(1,0)$ is given by

$J\left(E_{1}\right)=\left(\begin{array}{cc}m-1 & -p \\ 0 & q+e\end{array}\right)$.

As $\mathrm{m}<1$, one eigenvalue of $\mathrm{J}(\mathrm{E} 1)$ is positive and the other is negative. This indicates that $\mathrm{E} 1$ is a saddle point.

The equilibrium point $E_{2}(m, 0)$ has the Jacobian matrix $J\left(E_{2}\right)=\left(\begin{array}{cc}\mathrm{m}(1-\mathrm{m}) & -\frac{p}{m} \\ 0 & q+e\end{array}\right)$.

As $m<1$, both the eigenvalues of $J\left(E_{2}\right)$ are positive, and hence $E_{2}$ is always unstable.

The equilibrium point $E_{3}(0,1)$ has the Jacobian matrix

$$
J\left(E_{3}\right)=\left(\begin{array}{cc}
-m-\frac{p}{c} & 0 \\
\frac{q}{c} & -e \delta-\frac{q}{c}
\end{array}\right) .
$$

As both the eigenvalues of $J\left(E_{3}\right)$ are negative, $E_{3}$ is locally asymptotically stable.

Finally, we consider the stability issue of the most important equilibrium $E^{*}\left(x^{*}, y^{*}\right)$ :

We have the following Jacobian matrix at $E^{*}\left(x^{*}, y^{*}\right)$ : 
$\mathrm{J}\left(E^{*}\right)=\left(\begin{array}{ll}a_{11} & a_{12} \\ a_{21} & a_{22}\end{array}\right)$,

Where

$a_{11}=x^{*}\left(1+m-2 x^{*}\right)+\frac{p x^{*} y^{*}}{\left(c y^{*}+x^{*}\right)^{2}}$

$a_{12}=\frac{p x^{* 2}}{\left(c y^{*}+x^{*}\right)^{2}}$,

$a_{21}=\frac{q c y^{* 2}}{\left(c y^{*}+x^{*}\right)^{2}}$,

$a_{22}=-e \delta y^{* \delta}-\frac{q c y^{* 2}}{\left(c y^{*}+x^{*}\right)^{2}}$
The characteristic equation of $J\left(E_{3}\right)$ is

$\lambda^{2}+P \lambda+Q=0$

Where

$$
\begin{aligned}
& P=-\operatorname{trJ}\left(E^{*}\right)=-a_{11}-a_{22} \\
& Q=\operatorname{det} J\left(E^{*}\right)=a_{11} a_{22}-a_{12} a_{21}
\end{aligned}
$$

Then we have the following theorem guaranteeing the stability of $E^{*}\left(x^{*}, y^{*}\right)$.

Theorem 5.3 The necessary and sufficient condition for local asymptotic stability of the interior equilibrium $E^{*}\left(x^{*}\right.$, $\left.y^{*}\right)$ is that $P>0$ and $Q>0$.

It would be interesting if we can establish some sort of global behaviour of the interior equilibrium. Let $y>0$ and $\Omega$ $\left.=\left\{(x, y) \in \mathbf{R}^{2}: 0<x<1, y\right\rangle y\right\}$. Then we have the following theorem.

Theorem5.4 If $\mathrm{E}^{*}\left(\mathrm{x}^{*}, \mathrm{y}^{*}\right)$ is locally asymptotically stable with $c^{2} e(\delta+1) y^{\delta+2}>(2+m+e) c^{2} y^{2}+q$, then $E^{*}$ attracts all solutions of the system(2.2)lying in $\Omega$.

Proof . Let us write first equation of the system (2.2) as $\frac{d x}{d t}=P(x, y)$, and the second equation as $\frac{d y}{d t}=Q(x, y)$. Then for all $(x, y) \in \Omega$, we notice that

$\frac{\partial P}{\partial x}+\frac{\partial Q}{\partial x}=-3 x^{2}+2(1+m) x-m-\frac{p c y^{2}}{(c y+x)^{2}}+e-(1+\delta) y^{\delta}+\frac{q x^{2}}{(c y+x)^{2}}$

$\leq 2(1+m) x-m+e-e(1+\delta) y^{\delta}+\frac{q}{(c y)^{2}}$

$\leq \frac{1}{(c y)^{2}}\left[\left\{2+m+e-e(1+\delta) \underline{y}^{s}\right\}(c \underline{y})^{2}+q\right]<0$

$\frac{\partial P}{\partial x}+\frac{\partial Q}{\partial x}=-3 x^{2}+2(1+m) x-m-\frac{p(1+c y) y}{(1+b x+c y)^{2}}+e-(1+\delta) y^{\delta}+\frac{q x(1+b x)}{(1+b x+c y)^{2}}$

$\leq 2(1+m) x-m+e-e(1+\delta) y^{\delta}+q x(1+b x)$

$\leq 2+m+e-e(1+\delta) \underline{y}^{\delta}+q(1+b)<0$

Therefore, by Bendixson's criterion, there is no periodic orbit in $\Omega$. Hence the theorem follows from the Poinca'eBendixson theorem

\section{CONCLUDING REMARKS}

Predator-prey models are regarded as the building blocks of ecosystems. It is not so simple that the predator, being stronger species, will catch the prey and eat; and the prey will adopt many measures to trick the predator to avoid being hunted. Of course, the activities of both the prey and predator may be thought of as the struggle for their very existence, but this relationship does much more than this. Actually, predators have profound effects on an ecosystem and they play an important role in maintaining healthy prey populations.
Ever since the pioneering work of Lotka and Volterra, many mathematical models have been constructed to understand and analyze the predator-prey interaction. It is really impossible for any particular model to fit all natural subsystems, but there always has been a constant endeavour from researchers to find out the most suitable model. For this purpose, many modifications of the growth term have been made to incorporate various real-life phenomena. On the other hand, researchers have derived several functional responses, which may capture more realistic dynamics.

It was more than eighty years ago when the concept of Allee effect had drawn the attention of the scientists. Theoretical ecology remained silent for a long time on this issue. Recently, modellers have felt the necessity of modelling of Allee effect. Some models have been developed and cultured in last few years (for details, see [35]). In this paper, we have considered a predator-prey model where the prey is susceptible to Allee effect. The predator has a modified logistic growth and at the same time it consumes the prey following a Michaelis-Menten type ratio-dependent functional response. The number of parameters of the model has been reduced by suitable scaling. Then the dynamical behaviours of the resulting model (2.2) is studied. Existence and uniqueness of solutions of the model are discussed. It is shown (in Theorem 3.1 and Theorem 3.3) that the solutions of the system (2.2) remains non-negative forever, and they are uniformly bounded. These, in turn, imply that the system is biologically wellbehaved. We have derived some results on extinction of prey 
and predator. It is seen that if there is a very strong Allee effect, then it is almost impossible for the prey to survive. Also, an aggressive predator might cause extinction of the prey.

The stability analysis of the equilibrium points is very important from practical point of view. In this context, the interior equilibrium point is of utmost importance. Nowadays, preservation of ecological balance in nature is an issue which the entire globe is concerned with. Stability analysis of the interior equilibrium might provide the conditions for ecological balance in nature. Here we have presented the stability analysis of all the equilibrium points of the system (2.2). We have derived some global results also. The conditions of Theorems 5.3 and 5.4 may be used for ecological balance in nature.

\section{REFERENCES}

[1] P. Aguirre, E. Gonz'alez-Olivares, and E. S'aez,Two limit cycles in a Leslie-Gower predator-prey model with additive Allee effect, Nonlinear Anal. RWA, 10 (2009a), 14011416.

[2] P. Aguirre, E. Gonz'alez-Olivares, and E. S'aez, Three limit cycles in a Leslie-Gower predator-prey model with additive Allee effect, SIAM J. Appl. Math., 69 (2009b), 1244-1262.

[3] W. Allee, Animal aggregations, Quart. Rev. Biol., 2

(1927a), 367-398.

[4] W. Allee, Studies in animal aggregations: some physiological effects of aggregation on the brittle starfish, Ophioderma brevispina, J. Exp. Zool., 48 (1927b), 475495.

[5] W. Allee, Studies in animal aggregations: mass protection against colloidal silver among goldfishes, J. Exp. Zool., 61 (1932), 185-207.

[6] R. Arditi, and L. R. Ginzburg, Coupling in predator-prey dynamics : ratio- dependence, J. Theor. Biol., 139, (1989), 311-326.

[7] R. Arditi, and H. R. Akcakaya, Underestimation of mutual interference of predators, Oecologia, 83, (1990), 358-361.

[8] R. Arditi, J. M. Callois, Y. Tyutyunov and C. Jost, Does mutual interference always stabilize predator-prey dynamics? A comparison of models, C. R. Biologies, 327, (2004), 1037-1057.

[9] A.D. Bazykin, F.S. Berezovskaya, A.S. Isaev, and R.G. Khlebopros, Dynamics of forest insect density: bifurcation approach, J. Theor. Biol., 186 (1997), 267278.

[10] J.R. Beddington, Mutual interference between parasites or predators andits effecton searching efficiency, J. Animal Ecol., 44(1975), 331-340.

[11] S.P. Bera, A. Maiti, and G.P. Samanta, Dynamics of a food chain model with herd behaviour of the prey, Proceedings of the National Academy of Sciences, India Section A: Physical Sciences (to appear)

[12] L. Berec, E. Angulo, and F. Courchamp, Multiple allee effects and population man- agement, Trends Ecol. Evol., 22(2006), 185-191.

[13] F. Berezovskaya, G. Karev and R. Arditi, Parametric analysis of the ratio-dependent predator-prey model, $\mathrm{J}$.
Math. Biol., 43, (2001), 221-246.

[14] C.W. Clark, Mathematical Bioeconomic: The Optimal Mmanagement of Renewable Resources, Wiley, New York, 1990.

[15] C.W. Clark, The Worldwide Crisis in Fisheries: Economic Models and Human Be-havior, Cambridge University Press, Cambridge, 2007.

[16] T.H. Clutton-Brock, D. Gaynor, G.M. McIlrath, A.D.C. Maccoll, R. Kansky, P. Chadwick, M. Manser, J.D. Skinner, and P.N.M. Brotherton, Predation, group size and mortality in a cooperative mongoose, Suricata suricatta, J. Anim. Ecol., 68 (1999), 672-683.

[17] E.D. Conway, and J.A. Smoller, Globalanalysisofa system of predatorprey equations, SIAM J. Appl. Math., 46 (1986), 630-642.

[18] F. Courchamp, T. Clutton-Brock, and B. Grenfell, Inverse dependence and the Allee effect, Trends Ecol. Evol., 14 (1999), 405-410.

[19] F. Courchamp, L. Berec, and J. Gascoigne, Allee Effects in Ecology and Conservation, Oxford University Press, Oxford, 2008.

[20] D.L. DeAngelis, R.A. Goldstein, R.V. O’Neill, A model for trophic interaction, Ecology, 56(1975), 881-892.

[21] C.S. Elton, Animal Ecology, Sidgwick and Jackson,

London, 1927.

[22] J.D. Flores, J. Mena-Lorca, B. Gonz'alez-Yañez, and E. Gonz'alez-Olivares, Conse- quences of depensation in a Smith's bioeconomic model for open-access fishery, In R. Mondaini and R. Dilao (Eds.), Proceedings of international symposium on mathe- matical and computational biology (pp. 219232), E-papers Servios Editoriais Ltda, 2007.

[23] E. Gonz'alez-Olivares, and A. Rojas-Palma, Multiple Limit Cycles in a Gause type predator-prey model with Holling type III functional response and Allee effect on prey, Bull. Math. Biol., 73 (2011), 1378-1397.

[24] M. P. Hassell and G. C. Varley, New inductive population model for insect parasites and its bearing on biological control, Nature, 223, (1969), 1133-1137.

[25] C. S. Holling, The components of predation as revealed by a study of small mammal predation of the European pine sawfly, Can. Entomol., 91 (1959a), 293-320.

[26] C. S. Holling, Some characteristics of simple types of predation and parasitism, Can.

Entomol., 91 (1959b), 385-398.

[27] M. Kot, Elements of Mathematical Biology, Cambridge University Press, Cambridge, 2001.

[28] M. Liermann, and R. Hilborn, Depensation: evidence, modelsandimplications, Fish Fish., 2 (2001), 33-58.

[29] R.L. Lindeman, The trophic-dynamic aspect of ecology, Ecology 23 (1942), 399417. [30] A. Lotka, Elements of Physical Biology, Williams and Wilkins, Baltimore, 1925. [31] N. Macdonald, Biological Delay Systems: Linear Stability Theory, Cambridge University Press, Cambridge, 1989.

[32] A. Maiti, and G.P. Samanta, Deterministic and stochastic analysis of a prey-dependent predator-prey system, Internat. J. Math. Ed. Sci. Tech., 36 (2005), 6583.

[33] A. Maiti, B. Patra, and G. P. Samanta, Persistence and 
stability in a ratio-dependent predator-prey system with delay and harvesting, Natural Resource Modeling, 20 (2007), 575-600.

[34] A. Maiti, A. K. Pal, and G. P. Samanta, Effect of timedelay on a food chain model, Appl. Math. Comput., 200 (2008), 189-203.

[35] A. Maiti, P. Sen, D. Manna, and G.P. Samanta, A Predator-prey system with herd behaviour and strong Allee effect, Nonlinear Dynamics and Systems Theory (to appear).

[36] M.S. Mooring, T.A. Fitzpatrick, T.T. Nishihira, and D.D. Reisig, Vigilance, predation risk, and the Allee effect in desert bighorn sheep, J. Wildlife Management, 68 (2004), 519-532.

[37] J. D. Murray, Mathematical Biology, Springer-Verlag, New York, 1993. [38] E. Odum, Fundamentals of Ecology,

Saunders, Philadelphia, 1953.

[39] R. Pearl, and L. Reed, On the rate of growth of the population of the United States, Proc. Nat. Acad. Sci., 6 (1920), 275-288.

[40] P.W. Prince, C.E. Bouton, P. Gross, B.A. Mcpheropn, J.N. Thompson, and A.E. Weis, Interactions among three trophic levels: influence of plants on interactions between insect herbivores and natural enemies, Annu. Rev. Ecol. Syst., 11(1980), 41-65.

[41] F.J.Richards, A Flexible Growth Function for Empirical Use, Journal of Experimental Botany, 10 (1959), 290300.

[42] D.J. Rinella, M.S. Wipfli, C.A. Stricker, R.A. Heintz, and M.J. Rinella, Pacific salmon (Oncorhynchus sp.) runs and consumer fitness: growth and energy storage in stream-dwelling salmonids increase with salmon spawner density, Canad. J. Fish. Aquat. Sci, 69 (2012), 73-84.

[43] S. Ruan, and D. Xiao, Global analysis in a predator-prey system with nonmonotonic functional response, SIAM J. Appl. Math., 61 (2001), 1445-1472.

[44] P.A. Stephens, and W.J. Sutherland, Consequences of the Allee effect for behaviour, ecology and conservation TrendsEcol.Evol., 14(1999), 401-405.

[45] P.A. Stephens, W.J. Sutherland, and R.P. Freckleton, What is the Allee effect?, Oikos, 87 (1999), 185-190.

[46] G.A.K. van Voorn, L. Hemerik, M.P. Boer, and B.W. Kooi, Heteroclinic orbits in- dicate overexploitation in predator-prey systems with a strong Allee effect, Math. Biosci., 209 (2007), 451-469.

[47] P.F. Verhulst, Notice sur la loi que la population poursuit dans son accroissement, Correspondance math'ematique et physique, 10 (1838), 113-121.

[48] V. Volterra, Variazioni e fluttuazioni del numero di individui in specie animali con- viventi, Mem. Accd. Linc., 2 (1926), 31-113.

[49] G. Wang, X.G Liang, and F.Z Wang, The competitive dynamics ofpopulations subject to an Allee effect, Ecol. Model., 124 (1999), 183-192.

[50] M.H. Wang, and M. Kot, Speeds of invasion in a model with strong or weak Allee effects, Math. Biosci., 171 (2001), 83-97.

[51] J. Wang, J. Shi, and J. Wei, Predator-prey system with strong Allee effect in prey, J. Math. Biol., 62 (2011),
291-331.

[52]G. P. Samanta, and A. Maiti, Stochastic Gomatam model of interacting species: non-equilibrium fluctuation and stability, Systems Analysis Modelling Simulation, 43(6) (2003), 683-692.

[53] G. P. Samanta, D. Manna, and A. Maiti, Bionomic modelling of a three-species fishery with switching effect, Journal of Applied Mathematics and Computing, 12(1-2) (2003), 219-231.

[54] G. P. Samanta, and A. Maiti, Dynamical model of a single-species system in a polluted environment, Journal of Applied Mathematics and Computing, 16(12) (2004), 231-242.

[55]4. A. Maiti, and G. P. Samanta, Deterministic and stochastic analysis of a prey-dependent predator-prey system, International Journal of Mathematical Education in Science and Technology, 36(1) (2005), 65-83.

[56] A. Maiti, and G. P. Samanta, Complex dynamics of a food chain model with mixed selection of functional responses, Bulletin of the Calcutta Mathematical Society, 97(5) (2005), 393-412.

[57] A. Maiti, B. Patra, and G. P. Samanta, Persistence and stability of a food chain model with mixed selection of functional responses, Nonlinear Analysis : Modelling and Control, 11(2) (2006), 171-185.

[58] A. Maiti, and G. P. Samanta, Deterministic and stochastic analysis of a ratio-dependent prey-predator system, International Journal of Systems Science, 37(12) (2006), 817-826.

[59] A. Maiti, and G. P. Samanta, Rich dynamics of a timedelayed food chain model, Journal of Biological Systems, 14(3) (2006), 387-412.

[60] A. Maiti, B. Patra, and G. P. Samanta, Sterile insect release method as a control measure of insect pests : a mathematical model, Journal of Applied Mathematics and Computing, 22(3) (2006), 71-86.

[61] A. Maiti, M. M. Jana, and G. P. Samanta, Deterministic and stochastic analysis of a ratio-dependent predatorprey system with delay, Nonlinear Analysis : Modelling and Control, 12 (3) (2007), 383-398.

[62] A. Maiti, B. Patra, and G. P. Samanta, Persistence and stability in a ratio-dependent predator-prey system with delay and harvesting, Natural Resource Modelling, 20(4) (2007), 575-600.

[63] A. Maiti, A. K. Pal, and G. P. Samanta, Effect of timedelay on a food chain model, Applied Mathematics and Computation, 200(1) (2008), 189-203.

[64] A. Maiti, S. P. Bera, and G. P. Samanta A prey-predator model with microparasite infection in the predator, Journal of Biological Systems, 16(2) (2008), 219-239.

[65] A. Maiti, B. Patra, and G. P. Samanta, Bionomic exploitation of a ratio-dependent predator-prey system, International Journal of Mathematical Education in Science and Technology, 39(8) (2008), 1061-1076.

[66] A. Maiti, A. K. Pal, and G. P. Samanta, Usefulness of bio-control of pests in tea-a mathematical model, Mathematical Modelling of Natural Phenomena, 3(4) (2008), 96-113.

[67] S. Pathak, A. Maiti, and G. P. Samanta, Rich dynamics 
of a food chain model with Hassel-Varley type functional responses, Applied Mathematics and Computation, 208(2) (2009), 303-317.

[68] A. Maiti, and S. Pathak, A modified Holling-Tanner model in stochastic environment, Nonlinear Analysis : Modelling and Control, 14 (1) (2009), 51-71.

[69]B. Patra, A. Maiti, and G. P. Samanta, Effect of timedelay on a ratio-dependent food chain model, Nonlinear Analysis : Modelling and Control, 14 (2) (2009), 199216.

[70] A. Maiti, A. K. Pal, and G. P. Samanta, A nonlinear predator-prey system in deterministic and stochastic environment, International Journal of Ecological Economics \& Statistics, 16 (W10) (2010), 14-25.

[71]S. Pathak, A. Maiti, and G. P. Samanta, Rich dynamics of an SIR epidemic model, Nonlinear Analysis : Modelling and Control, 15 (1) (2010), 71-81.

[72] S. Pathak, and A. Maiti, Microbial pest control: a mathematical model, Journal of Biological Systems, 18(2) (2010), 455-478.

[73] S. Pathak, A. Maiti, and S. P. Bera, Effect of time-delay on a prey-predator model with microparasite infection in the predator, Journal of Biological Systems, 19(2) (2011), 365-387.

[74] S. Pathak, and A. Maiti, Pest control using virus as control agent: a mathematical model, Nonlinear Analysis: Modelling and Control, 17(1) (2012), 67-69.

[75] S. P. Bera, A. Maiti, and G. P. Samanta, Modelling herd behavior of prey: analysis of a prey-predator model, World Journal of Modelling and Simulation, 11(1) (2015), 3-14.

[76] S. P. Bera, A. Maiti, and G. P. Samanta, A delay dynamic model for HIV infected immune response, Journal of Applied Mathematics \& Informatics, 33 (5-6) (2015), 559-578.

[77] S. P. Bera, A. Maiti, and G. P. Samanta, A preypredator model with infection in both prey and predator, FILOMAT, 29(8) (2015), 1753-1767.

[78] S. P. Bera, A. Maiti, and G. P. Samanta, Stochastic analysis of a prey-predator model with herd behaviour of prey, Nonlinear Analysis: Modelling and Control, 21 (3) (2016), 345-361.

[79]A. Maiti, P.Sen, D. Manna, and G.P. Samanta, A predator-prey system with herd behaviour and strong Allee effect, Nonlinear Dynamics and Systems Theory, 16(1) (2016), 86-101.

[80] S. P. Bera, A. Maiti, and G. P. Samanta, Dynamics of a food chain model with herd behaviour of the prey, Model. Earth Syst. Environ., 2(131) (2016), DOI 10.1007/s40808-016-0189-4.

[81] A. Maiti, R. Paul, and S. Alam, A prey-predator model with Allee effect in prey and herd behaviour in both, Journal of Statistics and Mathematical Engineering, 2(1) (2016), 1-19.

[82]P.J. Pal, T. Saha, M. Sen , and M. Banerjee, A delayed predator-prey model with strong Allee effect in prey population growth, Nonlinear Dynamics, 68, 23-42.

[83] M. Frakas, Dynamical Models in Biology, Academic Press, New York, 2001 